\title{
Desenvolvimento do projeto mecânico conceitual de uma ferramenta didática para roleteamento cilíndrico ou plano
}

Anna Beatriz Oliveira Santos ${ }^{1}$, Dara Ferreira do Vale ${ }^{1}$, Henrique Braga de Freitas ${ }^{1}$, Magno da Silva Gomes ${ }^{1}$, Erick Ferreira da Silva Moura ${ }^{1}$, Richard Corrêa Esteves Junior ${ }^{1}$, Anderson Júnior dos Santos ${ }^{1}$

${ }^{1}$ Instituto Federal Sudeste de Minas Gerais Campus Muriaé, Muriaé MG, CEP 36884-036, Brasil. *e-mail: anderson.santos@ifsudestemg.edu.br

DOI: https://doi.org/10.30609/jeti.v4i04.13863

Abstract. In recent decades, the burnishing operation has gained relevance as a method of surface treatment of mechanical components. Burnishing is commonly used after the machining process in order to improve surface properties and resistance to cyclic loading of mechanical components. This article describes the development of the conceptual mechanical design of a burnishing tool with didactic purposes through Fusion 360 CAD (Computer Aided Design) software. In the development of the project, the steps of research, understanding of the problem and calculations necessary for the execution of the project were addressed, with all the results raised, the dimensioning of the helical compression spring and the screws used in the 3D modeling of the rolling tool was carried out. The project aimed to develop a reliable teaching tool that presents an alternative in relation to commercial equipment that have systems that use high pressure pumps or compressed air cylinders that increase the costs of the process. The results showed that the proposed burnishing tools have the capacity to withstand a burnishing force of $880 \mathrm{~N}$ and a burnishing pressure of $38.92 \mathrm{MPa}$ which presents the execution efficiency and applicability of the project when compared to commercial tools. In addition, the proposed project enables the construction and dissemination of the rolling technology.

Keywords: Burnishing; Mechanic project; CAD software; Tool; Fuison 360. 
Resumo. Nas últimas décadas, a operação de roleteamento tem ganhado relevância como um método de tratamento superficial de componentes mecânicos. O roleteamento é comumente utilizado após o processo de usinagem com objetivo de melhorar as propriedades da superfície e a resistência ao carregamento cíclico dos componentes mecânicos. O presente artigo descreve o desenvolvimento do projeto mecânico conceitual de uma ferramenta de roleteamento com finalidades didáticas por meio do software CAD (Desenho Assistido por Computador) Fusion 360. No desenvolvimento do projeto foram abordadas as etapas de pesquisa, entendimento do problema e cálculos necessários para execução do projeto, com todos os resultados levantados foi realizado o dimensionamento da mola helicoidal de compressão e dos parafusos utilizados no modelamento $3 \mathrm{D}$ da ferramenta de roleteamento. O projeto teve como finalidade desenvolver uma ferramenta didática confiável que apresente uma alternativa em relação aos equipamentos comerciais que apresentam sistemas que utilizam bombas de alta pressão ou cilindros de ar comprimido que elevam os custos do processo. Os resultados demonstraram que as ferramentas de roleteamento propostas tem a capacidade de suportar uma força de roleteamento de $880 \mathrm{~N}$ e uma pressão de roleteamento de 38,92 MPa o que apresenta a eficácia de execução e aplicabilidade do projeto quando comparada com ferramentas comerciais. Além disso, o projeto proposto viabiliza a construção e disseminação da tecnologia de roleteamento.

Palavras-chaves: Roleteamento, Projeto mecânico, Software CAD, Ferramenta, Fusion 360.

\section{Introdução}

Componentes mecânicos para máquinas-ferramentas, automóveis, aeronaves, moldes, matrizes de conformação, indústria biomédica e muitos outros elementos industriais são compostos de peças que normalmente requerem bom acabamento superficial e/ou maior resistência mecânica. Em decorrência dessas exigências, geralmente é necessário realizar tratamentos superficiais, como nitretação, cementação, jateamento e roleteamento, que modificam a superfície e melhoram o desempenho funcional do componente produzido [1].

Dentre as técnicas utilizadas para realizar o tratamento de superfícies de materiais metálicos, o roleteamento visa melhorar o acabamento de um componente a partir da deformação plástica da superfície sob condições de trabalho a frio [2]. Este processo, pode ser aplicado efetivamente em diversos setores industriais como, por exemplo, nos setores aeroespaciais e automotivo. De acordo com Figueiredo [3], componentes com superfícies lisas e polidas normalmente exibem vida em fadiga mais longa. 
Assim, o roleteamento (Fig. 1) destaca-se por apresentar os melhores resultados de rugosidade e por induzir tensões residuais de compressão, nas quais são benéficas à vida à fadiga [4]. Além disso, todos esses benefícios são obtidos sem alterar de maneira significativa a forma geométrica e a dimensão das peças [5].
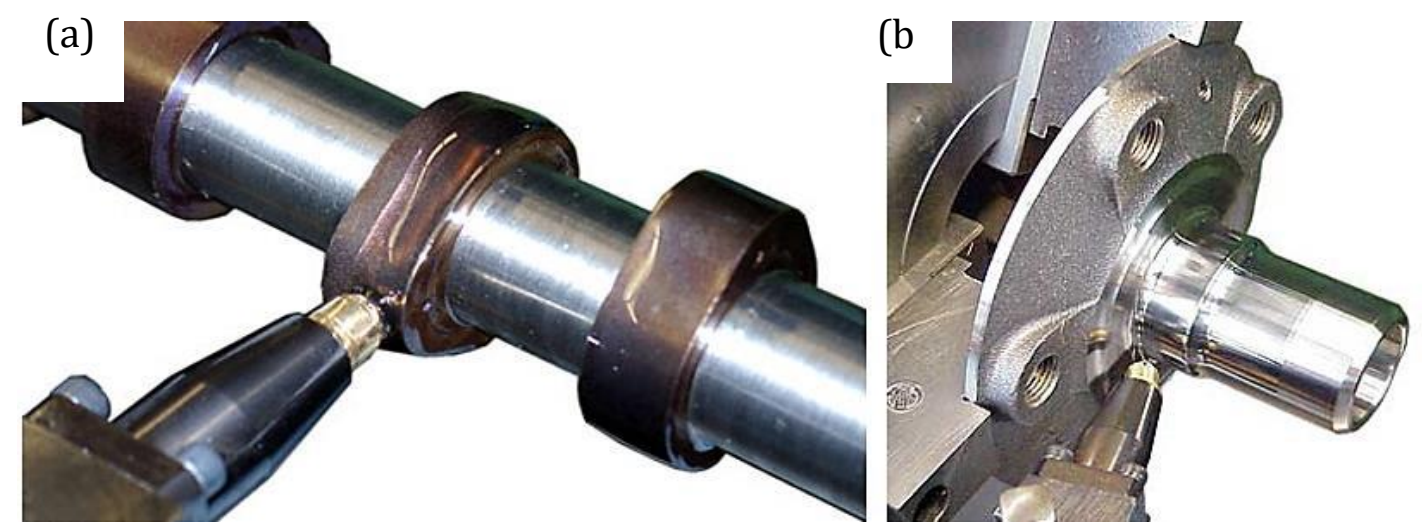

Figura 1. Aplicações do roleteamento. a) Roleteamento cilíndrico de um eixo virabrequim. b) Cubo de roda automotivo. Fonte: adaptado de Ecoroll [6].

El-Axir [7] indicou que o processo de roleteamento é comumente empregado após as operações convencionais de usinagem, sendo aplicado na própria máquina-ferramenta, onde a peça foi usinada. Desta maneira, a ferramenta para o roleteamento cilíndrico ou plano é fixada no porta-ferramenta da máquina, o que simplifica a aplicação, pois reduz significativamente o tempo, o custo e o número de etapas para se obter um componente com baixa rugosidade.

Em seus experimentos Figueiredo et al. 2021 [3] perceberam que a utilização do roleteamento reduziu em mais de $90 \%$ os valores de rugosidade em uma peça de aço ABNT 1020, comparados com os valores de rugosidade para as mesmas peças que passaram pela operação de torneamento. Leal [5] trabalhou com o aço ABNT 4140 e verificou que a redução da rugosidade foi da ordem de $80 \%$. Portanto, o roleteamento não produz apenas uma superfície com menor rugosidade, mas também aumenta a resistência à fadiga, à corrosão e ao desgaste.

Schuch et al. 2007 [8] investigaram a rugosidade, tensão residual e a contaminação da superfície de barras de diâmetro de $15 \mathrm{~mm}$ e comprimento de $100 \mathrm{~mm}$ fabricadas a partir de uma liga de TI-6Al-7Nb, para aplicações cirúrgicas, por meio de Microscopia Eletrônica de Varredura, Espectroscopia de Energia Dispersiva e Difração de Raios-X após o roleteamento cilíndrico. Os autores empregaram esferas com diâmetro de 4, 6 e 13 mm e pressão de trabalho entre 5 a $30 \mathrm{MPa}$. A velocidade de deslizamento manteve-se constante 
em $34 \mathrm{~m} / \mathrm{min}$, os menores valores de rugosidade foram alcançados para a pressão de $30 \mathrm{MPa}$ e para a esfera de diâmetro de $13 \mathrm{~mm}$, sendo encontrados valores de desvio médio aritmético $\left(R_{a}\right)$ de $0,1 \mu \mathrm{m}$ e altura total do perfil $\left(\mathrm{R}_{\mathrm{t}}\right)$ de $0,4 \mu \mathrm{m}$. Já para a tensão residual de compressão, identificaram-se os maiores valores, da ordem de $1150 \mathrm{Mpa}$, para a pressão de $30 \mathrm{MPa}$ e esfera de diâmetro de $6 \mathrm{~mm}$. No que diz respeito à contaminação da superfície, os resultados de Microscopia Eletrônica de Varredura e Espectroscopia de Energia Dispersiva das superfícies investigadas, não evidenciaram contaminação por partículas ou subprodutos do processo.

Apesar dos benefícios proporcionados pela operação de roleteamento, o investimento inicial para adquirir a ferramenta de roletear ainda é elevado, o que dificulta a aquisição do sistema de roletear [13]. Acrescentando-se que são encontrados poucos trabalhos na literatura, nos quais os pesquisadores desenvolvem suas próprias ferramentas para utilizar na operação de roleteamento. Desta maneira, este estudo desenvolveu o projeto conceitual de uma ferramenta didática de roleteamento cilíndrico ou plano para ser utilizada em um torno e fresadora convencional ou em um torno e centro de usinagem com comando numérico computadorizado (CNC).

\section{Materiais e métodos}

Para o desenvolvimento da ferramenta de roleteamento foi utilizado o software CAD Fusion 360. De acordo com Junk et al. 2021 [9], o Fusion 360 é um programa CAD da empresa Autodesk que combina projeto e modelagem 3D, simulação, projeto generativo, documentação, colaboração e manufatura em um único ambiente integrado.

Desta maneira, a metodologia do projeto foi baseada em estudos sobre o tema e, a partir disso, o mesmo foi dividido em duas etapas. A etapa I refere-se ao modelamento e dimensionamento da ferramenta de roleteamento empregando uma esfera como elemento rolante. A etapa II refere-se ao modelamento e dimensionamento da ferramenta de roleteamento empregando um rolete como elemento rolante. Salienta-se também que, foi modelada uma geometria adequada para que a ferramenta possa ser fixada no porta ferramenta de um torno convencional, fresadora convencional, torno $\mathrm{CNC}$ e centro de usinagem CNC de três eixos.

Para o projeto de ambas as ferramentas de roleteamento foi necessário calcular a força de roleteamento envolvida no processo. A força de roleteamento foi baseada na 
constante e na deformação elástica da mola helicoidal de compressão (lei de Hooke), conforme apresentado na Eq. (1).

$F_{N}=K \cdot \Delta x$

\section{Onde:}

$\mathrm{F}_{\mathrm{N}}=$ Força de roleteamento $[\mathrm{N}]$;

$\mathrm{K}=$ constante elástica $[\mathrm{N} / \mathrm{mm}]$;

$\Delta \mathrm{x}=$ deformação elástica $[\mathrm{mm}]$;

Para uma compreensão abrangente da força de roleteamento que atua durante o processo foi elaborado um desenho esquemático simplificado, conforme a Fig. (2).

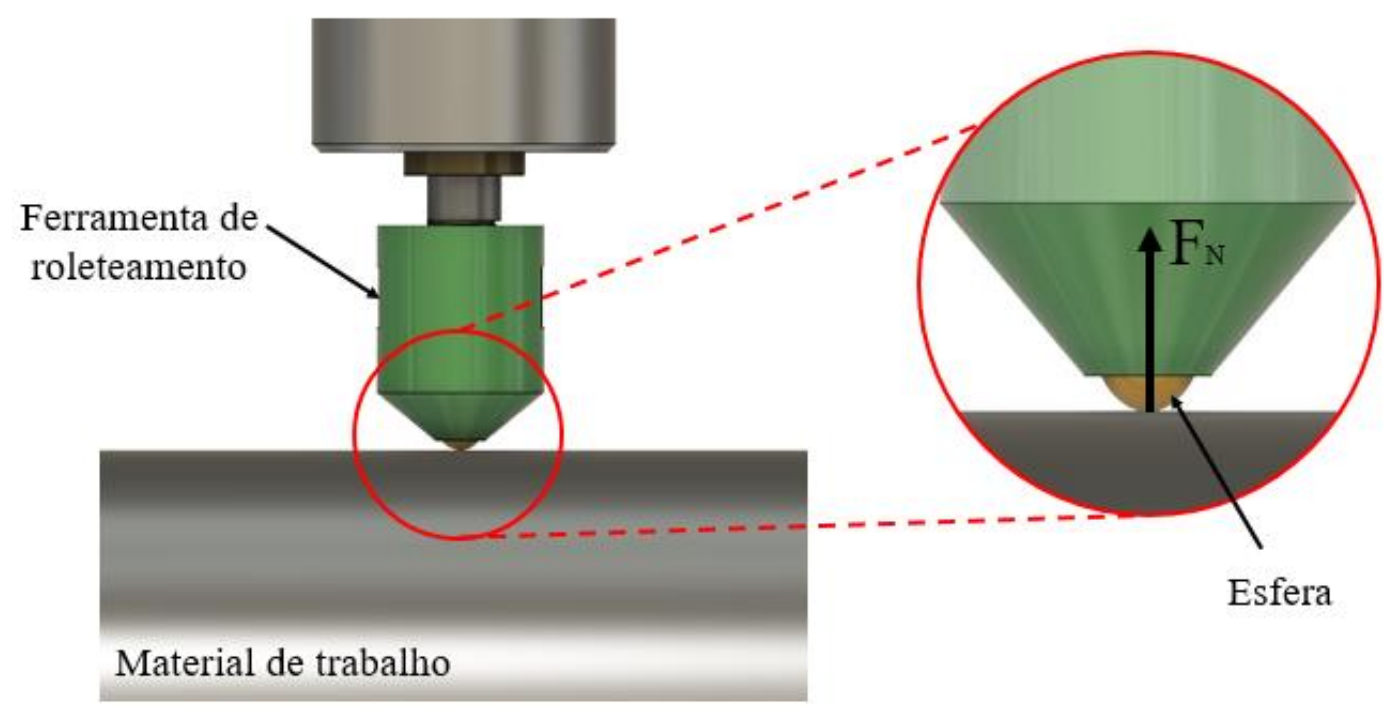

Figura 2. Força de roleteamento no contanto entre a esfera e a superfície do material de trabalho.

A pressão máxima suportada pela ferramenta foi estimada baseando-se na teoria do contato de Hertz. Onde, considera-se o contato entre duas superfícies e também a direção de carregamento aplicado. Deste modo, o modelo de MaiB [10] apresentado na Eq. (2) estima a força de roleteamento em função da pressão e da área de roleteamento.

$F_{N}=P_{w} \cdot\left(\frac{d_{b}}{2}\right)^{2} \cdot \pi \cdot n$

\section{Onde:}

$\mathrm{F}_{\mathrm{N}}=$ força de roleteamento $[\mathrm{N}]$;

$\mathrm{Pw}=$ pressão $[\mathrm{MPa}]$

$\mathrm{d}_{\mathrm{b}}=$ diâmetro da esfera $[\mathrm{mm}]$; 
$\mathrm{n}=$ coeficiente de eficiência, do qual pode ser assumido como 0,8 como observado por Denkena [11].

Portanto, para estimar a pressão de trabalho da ferramenta de roleteamento foi realizado a equivalência entre a força de roleteamento da Eq. (1) e a força de roleteamento do modelo de Maib [10] apresentado na Eq. (2), obtendo-se a Eq. (3).

$P_{w}=\frac{\Delta x \cdot K}{\left(\frac{d b}{2}\right)^{2} \cdot \pi \cdot n}$

Além disso, foi necessário calcular a tensão máxima suportada pelos parafusos do tipo Allen determinados para o projeto da ferramenta de roleteamento. Para determinar a tensão máxima foi necessário determinar à área por meio do diâmetro menor do parafuso. Sendo assim, foi empregado a Eq. (4) para calcular o diâmetro menor do parafuso, Eq. (5) para calcular a área e a Eq. (6) para determinar a tensão aplicada no parafuso. A tenção de cisalhamento, Eq. (8) e Eq. (9), atuante no eixo rolete do conjunto também foi calculada.

$d=D-1,23 \cdot P$

$A=\frac{\pi \cdot d^{2}}{4}$

$F=\frac{F_{N}}{q}$

$\sigma=\frac{F}{A}$

$\tau=0,75 \cdot \sigma$

$\tau=\frac{V}{A}$

\section{Onde:}

d= diâmetro menor do parafuso [mm];

$\mathrm{D}=$ Diâmetro da rosca $[\mathrm{mm}]$;

$\mathrm{P}=$ passo da rosca $[\mathrm{mm}]$;

$\mathrm{A}=$ área da seção transversal do parafuso $\left[\mathrm{mm}^{2}\right]$;

$\mathrm{F}=$ Força $[\mathrm{N}]$;

$\mathrm{q}=$ quantidade de parafusos [admissional];

$\sigma=$ Tensão $[\mathrm{MPa}]$;

$\mathrm{t}=$ Tensão de cisalhamento [Mpa]; 
$\mathrm{V}=$ Força cortante $[\mathrm{N}]$.

\section{Resultados e discussão}

\subsection{Ferramenta de roleteamento empregando esfera}

A Fig. (3) está ilustrada a vista explodida (etapa I) da ferramenta de roleteamento empregando uma esfera como elemento rolante. Com este arranjo, a esfera gira com a rotação ou deslocamento da peça de trabalho devido a força de atrito envolvida no contato entre a esfera e o material de trabalho. Nesta ferramenta, quase todas as peças foram projetadas e modeladas de forma a serem obtidas pelo processo de usinagem, salvo algumas exceções como, mola, parafusos e esferas, que podem ser adquiridos facilmente no comércio.

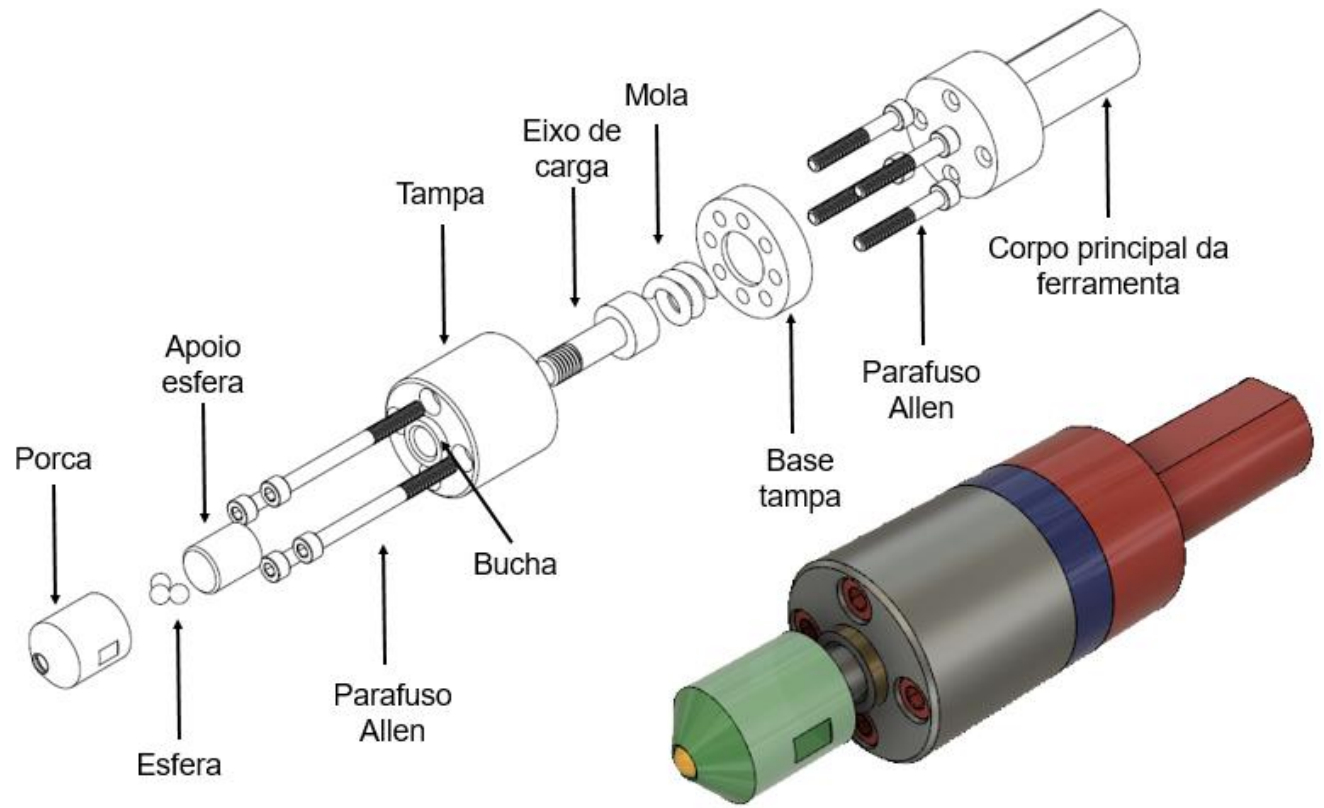

Figura 3. Ferramenta de roleteamento empregando uma esfera como elemento rolante.

A ferramenta de roleteamento apresentada na Fig. (3) é constituída por:

Corpo principal da ferramenta - Tem como principal objetivo fixar a ferramenta no porta-ferramenta do torno, fresadora ou centro de usinagem CNC. Além disso, confere rigidez a ferramenta e proporciona sustentação aos demais componentes.

Base tampa - Suporta a pressão exercida pela mola.

Mola - A mola helicoidal de compressão tem a finalidade de armazenar a energia mecânica e assumir a deformação elástica quando submetida a força de roleteamento. Sendo assim, foi determinado uma mola helicoidal comercial com constante elástica de $80 \mathrm{~N} / \mathrm{mm}$ e deslocamento máximo de $11 \mathrm{~mm}$.

Journal of Experimental and Techniques Instrumentation - JETI, v.4, n.04, 2021 
Eixo de carga - É o responsável por aplicar a força de roleteamento (Eq. (1)) por meio do deslocamento da mola helicoidal de compressão.

$$
\begin{aligned}
& F_{N}=80\left[\frac{\mathrm{N}}{\mathrm{mm}}\right] \cdot 11[\mathrm{~mm}] \\
& \mathrm{F}_{\mathrm{N}}=880 \mathrm{~N}
\end{aligned}
$$

Portanto, o eixo de carga aplica uma pressão de roleteamento de 38,92 $\mathrm{MPa}$, conforme a Eq. (3).

$$
P_{w}=\frac{80.11}{\left(\frac{6}{2}\right)^{2} \cdot \pi \cdot 0,8}
$$

$\mathrm{P}_{\mathrm{w}}=38,92 \mathrm{MPa}$

Tampa - A tampa tem como finalidade proteger o eixo de carga e a mola do ambiente externo. Além disso, suporta os esforços decorrentes do processo.

Bucha - Sustentar e possibilitar o movimento deslizante do eixo de carga com menor atrito possível.

Parafuso Allen - Tem como finalidade fixar a tampa na base da tampa e suportar os esforços solicitantes envolvidos durante o processo de roleteamento. Para todo o sistema optou-se por oito parafusos forjados a quente, sendo o M5 x 0,8 de classe 5.8, ou seja, com resistência à tração de $540 \mathrm{MPa}$ e limite de escoamento de $420 \mathrm{MPa}$. Portanto, dividindo a força máxima de roleteamento de $880 \mathrm{~N}$ por 8 parafusos, foi encontrada uma força de 110 $\mathrm{N}$, ou seja, cada parafuso deve suportar uma carga de $110 \mathrm{~N}$ no regime elástico. Sendo assim, foi necessário determinar a tensão máxima suportada por cada parafuso, conforme as Eq. (4), (5), (6) e (7).

$d=5-1,23 \cdot 0,8$

$d=4,016 \mathrm{~mm}$

$A=\frac{\pi \cdot 4,016^{2}}{4}$

$A=12,66 \mathrm{~mm}^{2}$

$F=\frac{880[N]}{8}$

$F=110[N]$ 
$\sigma=\frac{110[\mathrm{~N}]}{12,66\left[\mathrm{~mm}^{2}\right]}$

$\sigma=8,69 \mathrm{MPa}$

Fundamentado no resultado de 8,69 MPa referente ao cálculo de tensão suportada por cada parafuso, pode-se verificar que, os parafusos determinados para compor o projeto são capazes de suportar os esforços solicitantes da ferramenta de roleteamento sem alcançar a tensão de escoamento de $420 \mathrm{MPa}$ do material. Os parafusos determinados apresentam uma tensão de escoamento de, aproximadamente, 48 vezes maior que o necessário para o projeto da ferramenta. Sendo assim, a escolha dos parafusos deve-se as dimensões da ferramenta de roleteamento e por tais parafusos serem comerciais.

Apoio esfera - superfície de deslizamento e apoio das esferas. Este componente é rosqueado no eixo de carga e na porca. Portanto, pode ser substituído por desgaste ou por opção de mudar a configuração do elemento rolante.

Esferas - a ferramenta é constituída de três esferas com diâmetro de $6 \mathrm{~mm}$. Sendo que, duas esferas são de apoio e circulam continuamente em condições dinâmicas com o objetivo de suportar as cargas aplicadas. Em uma terceira esfera há um contato com o material de trabalho. Estas esferas serão lubrificadas pelo jato de fluido de corte através do sistema de lubri-refrigeração original das próprias máquinas-ferramentas.

Porca - tem a função de fixar e permitir a remoção das esferas para limpeza, reajuste e substituição em caso de desgaste.

A estrutura da ferramenta de roleteamento foi projetada em aço, buscando propriedades como ductilidade, resiliência e resistência mecânica. Estas propriedades são relevantes, pois toda estrutura da ferramenta não deve sofrer deformações plásticas permanentes. Sendo assim, a ferramenta foi projetada para trabalhar dentro do regime elástico.

$\mathrm{Na}$ Tabela 1 foram sumarizados os materiais empregados no projeto juntamente com as justificativas de escolhas baseado em resistência mecânica, fabricação, atrito e custo. Os materiais empregados devem possuir propriedades mecânicas suficiente para suportar os esforços solicitantes, não permitindo que ocorram danos a ferramenta durante o processo de roleteamento. Baseado nas condições nas quais a estrutura é solicitada, optou-se pela utilização de aço com baixo e médio teor de carbono. 
Tabela 1. Apresentação dos componentes, material e justificativas para a ferramenta de roleteamento empregando esfera.

\begin{tabular}{|c|c|c|}
\hline Componente & Material empregado & Justificativa \\
\hline Porca & $\begin{array}{c}\text { Aço ABNT } 1045 \\
\text { temperado e revenido - } 302 \\
\text { HV }\end{array}$ & $\begin{array}{c}\text { Resistência mecânica e menor } \\
\text { desgaste }\end{array}$ \\
\hline Esfera & Metal duro & $\begin{array}{c}\text { Resistência mecânica e menor } \\
\text { desgaste }\end{array}$ \\
\hline Apoio esfera & $\begin{array}{l}\text { Aço ABNT } 1045 \\
\text { temperado e revenido }\end{array}$ & Resistência mecânica \\
\hline Bucha & Bronze TM-23 & $\begin{array}{c}\text { Atrito - Serve de apoio para o eixo } \\
\text { de carga e diminui o atrito. }\end{array}$ \\
\hline Tampa & $\begin{array}{l}\text { Aço ABNT } 1045 \\
\text { temperado e revenido }\end{array}$ & Resistência mecânica \\
\hline Eixo de carga & $\begin{array}{l}\text { Aço ABNT } 1045 \\
\text { temperado e revenido }\end{array}$ & Resistência mecânica \\
\hline Mola & $\begin{array}{l}\text { Aço mola (SAE 6150) } \\
\text { temperado e revenido }\end{array}$ & $\begin{array}{l}\text { Mola comercial especificada de } \\
\text { acordo com a pressão de trabalho. }\end{array}$ \\
\hline Base tampa & $\begin{array}{l}\text { Aço ABNT } 1045 \\
\text { temperado e revenido }\end{array}$ & Resistência mecânica \\
\hline $\begin{array}{l}\text { Corpo principal da } \\
\text { ferramenta }\end{array}$ & Aço ABNT 1020 & Fabricação e custo \\
\hline Parafuso Allen & Aço forjado à quente & Resistência e custo \\
\hline
\end{tabular}

\subsection{Ferramenta de roleteamento empregando rolete}

A Fig. (4) apresenta a vista explodida (etapa 2) da ferramenta de roleteamento empregando um rolete rígido como elemento rolante. Esta ferramenta possui um arranjo semelhante ao apresentado na Fig. (3) porém, com a modificação do elemento rolante. Sendo assim, a ferramenta apresenta um cabeçote rosqueado ao eixo de carga que pode ser substituído ou alternado por outras geometrias de elementos rolantes, composto pelo suporte rolete, eixo rolete, rolete, capa e parafusos Allen. 


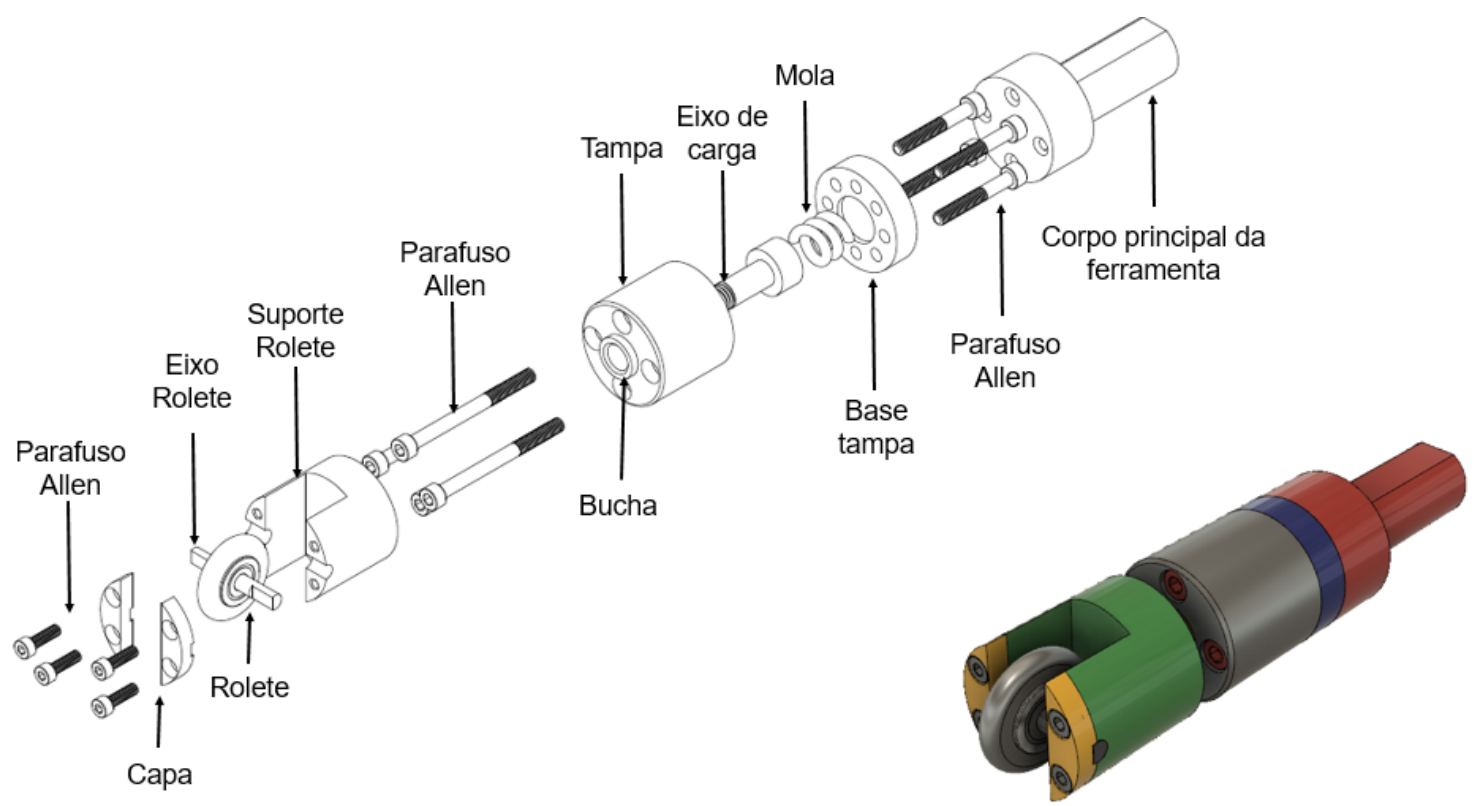

Figura 4. Ferramenta de roleteamento empregando rolete como elemento rolante.

Desta forma, os componentes que foram adicionados ao conjunto para formar o cabeçote da ferramenta de roleteamento com rolete rígido são:

Rolete rígido - Sua principal função é realizar a deformação plástica a frio da superfície do material de trabalho. Sendo que, quando em contato com a superfície de trabalho deve suportar as tensões de contato sem a ocorrência de deformação plástica no material do rolete.

Suporte rolete - é um componente estrutural para fixar o eixo rolete, cuja função é servir de apoio e suportar os esforços mecânicos envolvidos no processo de roleteamento.

Eixo rolete - é um componente de acoplamento entre o suporte rolete e o rolete rígido. Sua principal função é guiar o movimento de rotação do rolete rígido quando em contato com a superfície do material de trabalho. Tem a mesma dimensão do furo do rolete, ou seja, $6 \mathrm{~mm}$ para este projeto.

O eixo rolete está sujeito à um esforço de cisalhamento duplo. Esse componente será fabricado de Aço ABNT 1045 temperado e revenido e, de acordo com Gallo [12], o valor da tensão de escoamento para esse material é de $544 \mathrm{MPa}$. Utilizando a Eq. (8) pode-se determinar a tensão de cisalhamento admissível para esse caso.

$$
\begin{gathered}
\tau=0,75 \cdot 544 \\
\tau=408 \mathrm{MPa}
\end{gathered}
$$


Ou seja, para trabalhar dentro do regime elástico, a tensão de cisalhamento atuante sobre o eixo rolete deve ser menor que $408 \mathrm{MPa}$.

Considerando a força máxima de roleteamento de $880 \mathrm{~N}$, encontrada através da Equação 1, tem-se uma força cortante de 440 N. Através das Eq. (5) e (9) determina-se a tensão de cisalhamento atuante nesse projeto.

$$
\begin{aligned}
A & =\sqrt{\frac{6 \cdot 4}{\pi}} \\
A & =2,76 \mathrm{~mm}^{2} \\
\tau & =\frac{440}{2,76} \\
\tau & =159 \mathrm{MPa}
\end{aligned}
$$

Pelo que foi exposto no cálculo, a tensão de cisalhamento de $159 \mathrm{MPa}$ atuante no eixo rolete é cerca de duas vezes e meia menor que a tensão de cisalhamento admissível de $408 \mathrm{MPa}$. Logo, componente irá suportar o esforço durante o trabalho.

Capa - componente de fixação entre o eixo rolete e o suporte rolete.

Parafuso Allen - Realizar a união entre a capa, eixo rolete e suporte rolete.

A Tabela 2 apresenta os materiais escolhidos para os componentes do cabeçote rosqueado.

Tabela 2. Apresenta os componentes, material empregado e justificativas para o cabeçote de roleteamento empregando rolete rígido.

\begin{tabular}{c|c|c}
\hline Componente & Material empregado & Justificativa \\
\hline Suporte rolete & Aço ABNT 1020 & Fabricação e custo \\
Eixo rolete & Aço ABNT 1045 temperado e & Resistência mecânica \\
& revenido & Resistência mecânica e menor \\
Rolete & Aço SAE 52100 temperado e & desgaste \\
Capa & revenido & Resistência e custo \\
Parafuso & Aço ABNT 1020 & Resistência e custo \\
Allen & Aço forjado a quente & \\
\hline
\end{tabular}

3.3 Correlação entre as ferramentas projetadas e as ferramentas comerciais

A empresa Ecoroll 2021 [6] fabricante de equipamentos de roleteamento comumente apresenta três tipos de sistemas para roleteamento:

$1^{\circ}$ - Sistema hidráulico - geralmente composto por bombas com pressões entre 0 $45 \mathrm{MPa}$, motor elétrico, mangueiras, fluido de corte e ferramenta de roleteamento. Este 
sistema apresenta como finalidade o roleteamento de materiais com durezas de até $832 \mathrm{HV}$ e roleteamento de superfícies complexas. Além disso, este sistema induz maiores tensões residuais de compressão em relação aos sistemas com ar comprimido e mola de compressão.

$2^{\circ}$ Ar comprimido - geralmente constituídos por cilindros de ar comprimido, mangueiras, manômetros e ferramenta de roleteamento. Este sistema apresenta como característica a não contaminação do ambiente e a capacidade de realizar roleteamento em materiais com dureza máxima de 595 HV.

$3^{\circ}$ Mola de compressão - essa ferramenta é constituída por uma estrutura rígida e uma seção flexível com mola de compressão que aplica a tensão de contato na superfície do material por meio de um elemento rolante. Este sistema apresenta capacidade de realizar roleteamento em materiais com dureza máxima de $446 \mathrm{HV}$ e dentre os sistemas apresentados é o que apresenta menor capacidade de induzir tensões residuais de compressão, sendo mais indicado para operações de acabamento superficial em superfícies com geometrias simples ou complexas. Essa ferramenta assemelha-se com o projeto proposto.

Quando comparado as ferramentas de roleteamento projetadas neste trabalho com as ferramentas fornecidas pela empresa Ecoroll 2021, percebe-se que as ferramentas projetas são constituídas por uma mola helicoidal de compressão e podem ser fabricadas pelo processo de usinagem, ou seja, as ferramentas projetadas podem ser elaboradas pelos usuários e utilizadas como recurso didático para aulas de laboratório ou instrumento de pesquisa para investigar o processo de roleteamento. Sendo que, a ferramenta projetada é recomendada para operações de acabamento em materiais com dureza máxima de $446 \mathrm{HV}$.

Portanto, a principal característica das ferramentas projetadas em relação as ferramentas comerciais é o menor custo de aquisição pois, pode ser desenvolvida a partir do projeto proposto e pelo processo de usinagem. Viabilizando a disseminação da tecnologia principalmente na comunidade acadêmica.

\section{Conclusão}

Disseminar o processo de roleteamento é de fundamental importância para o desenvolvimento e melhoria dos produtos usinados.

Os resultados demonstraram que o projeto das ferramentas de roleteamento propostas, apresentam uma capacidade de suportar uma força de roleteamento máxima de $880 \mathrm{~N}$ e uma pressão de roleteamento máxima de 38,92 MPa para a mola helicoidal de compressão especificada. Dessa forma, apresenta uma eficácia de execução e aplicabilidade do projeto quando comparada com ferramentas comerciais. 
O projeto conceitual da ferramenta didática de roleteamento proposto, apresenta uma alternativa viável para execução em aulas, nas disciplinas de processo de fabricação ou pesquisas sobre roleteamento. Sendo que, as ferramentas projetadas podem ser aplicadas em torno convencional, torno ou centro de usinagem CNC de três eixos.

O projeto também apresenta como alternativa a possibilidade de utilizar elementos rolantes com diversas geometrias. Sendo que, a alternância entre os elementos rolantes pode ser realizada por meio da troca do cabeçote de roleteamento. Essa característica possibilita a diversificação do elemento rolante de acordo com a geometria da peça de trabalho.

\section{Agradecimentos}

O presente trabalho foi realizado com apoio do Conselho Nacional de Desenvolvimento Científico e Tecnológico - CNPq, por meio do programa Institucional de Bolsas de Iniciação Científica - PIBIC - edital 05/2020 - PROPPI - Processo - n²3223.002421/2020-16.

\section{Bibliografia}

[1] Travieso-Rodriguez, J. A., Gomez-Gras, G., Dessein, G., Carrillo, F., Alexis, J., JorbaPeiro, J., \& Aubazac, N. (2015). Effects of a ball-burnishing process assisted by vibrations in G10380 steel specimens. The International Journal of Advanced Manufacturing Technology, 81(9), 1757-1765.

[2] Cangussu, V. M., Abrão, A. M., Magalhães, F. D. C., Denkena, B., Breidenstein, B., \& Meyer, K. (2020). Modelagem e análise numéricas da operação de roleteamento do aço ABNT 4140. Matéria (Rio de Janeiro), 25.

[3] Figueiredo dos Santos, F., da Costa Silva, S., Abrão, A. M., Denkena, B., Breidenstein, B., \& Meyer, K. (2021). Influence of the Carbon Content on the Surface Integrity of Deep Rolled Steels. Journal of Tribology, 143(8), 081702.

[4] Duncheva, G. V., Maximov, J. T., Dunchev, V. P., Anchev, A. P., Atanasov, T. P., \& Capek, J. (2020). Single toroidal roller burnishing of 2024-T3 Al alloy implemented as mixed burnishing process. The International Journal of Advanced Manufacturing Technology, 111(11), 3559-3570.

[5] Leal, C. A. A. (2020). Estudo sobre o roleteamento do aço ABNT 4140 temperado e revenido.

[6] Ecoroll. Ecoroll suface matters, 2021. Disponível em: < https://www.ecoroll.de/en/products.html>. Acesso em 24 de julho de 2021. 
[7] El-Axir, M. H. (2007). An investigation into the ball burnishing of aluminium alloy 6061T6. Proceedings of the Institution of Mechanical Engineers, Part B: Journal of Engineering Manufacture, 221(12), 1733-1742.

[8] Schuh, A., Zeller, C., Holzwarth, U., Kachler, W., Wilcke, G., Zeiler, G., ... \& Bigoney, J. (2007). Deep rolling of titanium rods for application in modular total hip arthroplasty. Journal of Biomedical Materials Research Part B: Applied Biomaterials: An Official Journal of The Society for Biomaterials, The Japanese Society for Biomaterials, and The Australian Society for Biomaterials and the Korean Society for Biomaterials, 81(2), 330335.

[9] Junk, S., \& Burkart, L. (2021). Comparison of CAD systems for generative design for use with additive manufacturing. Procedia CIRP, 100, 577-582.

[10] Maiß, O. (2019). Lebensdauererhöhung von Wälzlagern durch mechanische Bearbeitung. TEWISS-Technik und Wissen GmbH.

[11] Denkena, B., Abrão, A., Krödel, A., \& Meyer, K. (2020). Analytic roughness prediction by deep rolling. Production Engineering, 14(3), 345-354.

[12] Gallo, G. B. (2006). Influência do tratamento térmico sobre a tenacidade de um aço AISI SAE 1045 com médio teor de carbono, avaliada por ensaios de impacto. Dissertação, Faculdade de Engenharia do Campus de Guaratinguetá, Universidade Estadual Paulista.Junk, S., \& Burkart, L. (2021). Comparison of CAD systems for generative design for use with additive manufacturing. Procedia CIRP, 100, 577-582.

[13] Jerez-Mesa, R., Travieso-Rodriguez, J. A., Gomez-Gras, G., \& Lluma-Fuentes, J. (2018). Development, characterization and test of an ultrasonic vibration-assisted ball burnishing tool. Journal of Materials Processing Technology, 257, 203-212. 